\title{
Frequency of Psychosomatic Symptoms in Patients with Covid-19
}

\author{
Pir Hossein Kolivand ${ }^{1,2 *}$, Zahra Kolivand ${ }^{3}$, Hadi Kazemi ${ }^{4}$, Maryam Kolivand ${ }^{5}$ \\ ${ }^{1}$ Health Economic Department, Shahed University, Tehran, Iran \\ ${ }^{2}$ Shefa Neuroscience Research Center, Khatam Alanbia Hospital, Tehran, Iran \\ ${ }^{3}$ School of Medicine, Tehran University of Medical Sciences, Tehran, Iran \\ ${ }^{4}$ School of Medicine, Shahed University, Tehran, Iran \\ ${ }^{5}$ School of Medicine, Shahid Beheshti University of Medical Sciences, Tehran, Iran
}

Article Info:

\section{A BSTRACT}

Introduction: Infection with SARS-CoV-2 spread rapidly around the world and is recognized by the World Health Organization as a pandemic. Psychological disorders, such as anxiety, have caused countless problems for societies during the last years. In the present study, various clinical features of patients with COVID-19 disease, particularly psychosomatic symptoms, were investigated. Materials and Methods: Clinical characteristics of 2928 patients with SARS$\mathrm{CoV}-2$ infection, including risk factors such as diabetes mellitus, hypertension, cardiovascular disease, and chronic kidney disease as well as anxiety were collected in different cities in Iran (Tehran, Karaj, Hamedan, and Kermanshah) and analyzed. Results: Our data have shown different patterns of clinical features and anxiety rates in patients with COVID-19 in various regions of Iran. However, the characteristics of chronic inflammatory lung disease were similar in these patients in various areas. Conclusion: Geographical factors, as well as the medical facilities of the participants, may affect the clinical features of patients with COVID-19 disease.

*Corresponding Author: Pir Hossein Kolivand

Email: peirhossein@yahoo.com 
فراوانى علائم اختلال روان تنى (سايكوسوماتيك) در بيماران مبتلا به كوويد - 19

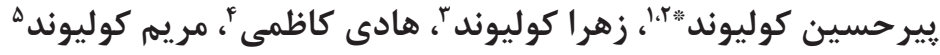

اكروه اقتصاد سلامت، دانشكاه شاهد، تهران، ايران

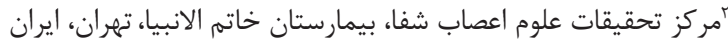

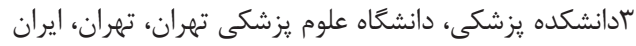

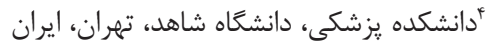

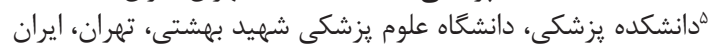

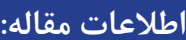

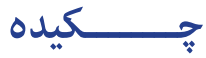

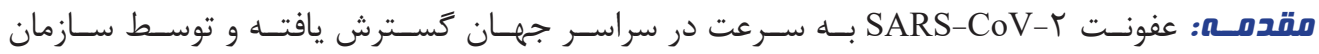

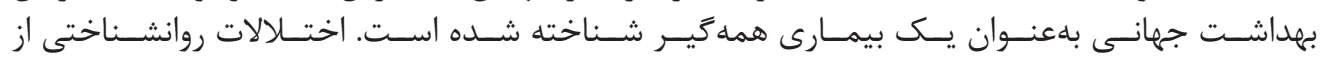

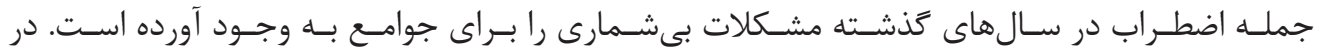

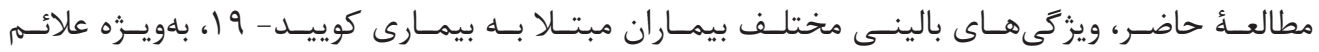

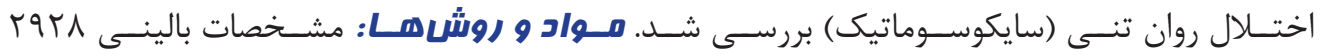

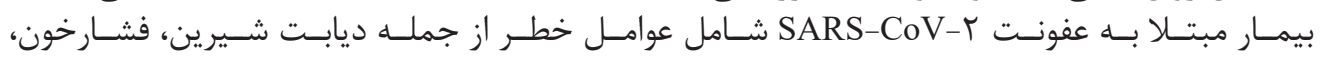

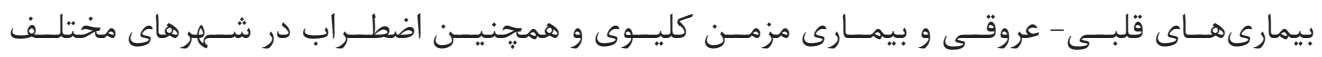

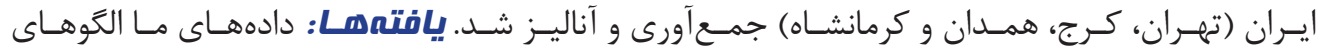

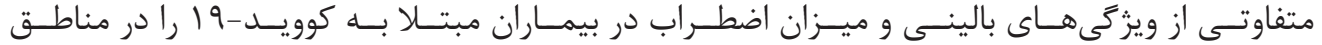

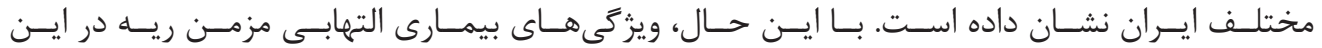

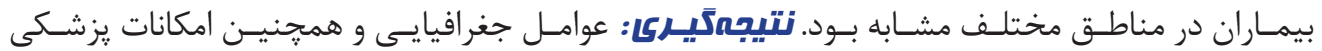

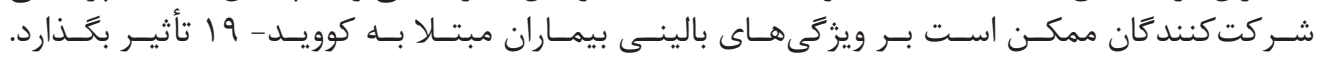

وازههاى كليدى:

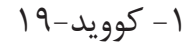
r- بإيروس كرونا r بإندمى

$$
\text { *نويسنده مسئول: پِيرحسين كوليوند }
$$

peirhossein@yahoo.com يست الكترونيك 


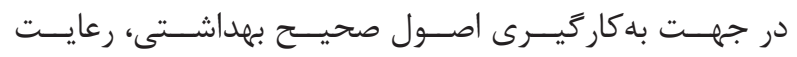

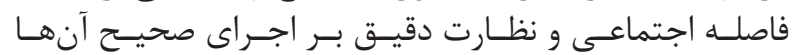

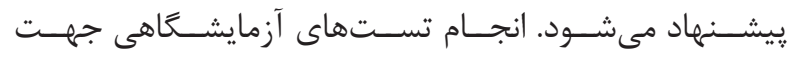

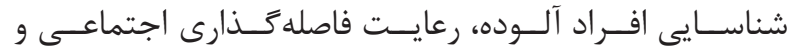

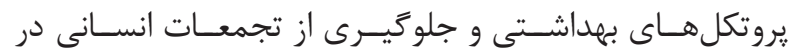

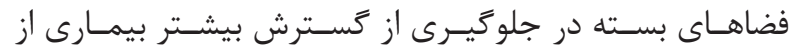

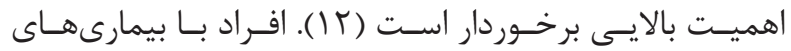

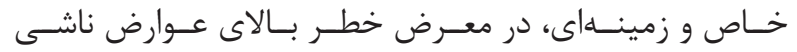

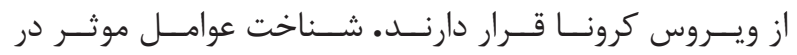

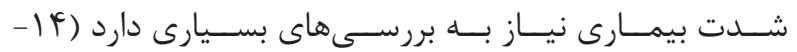

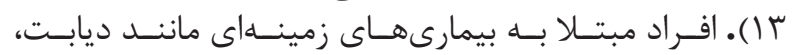

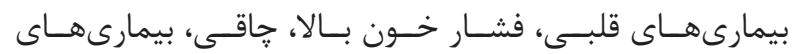

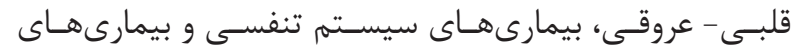

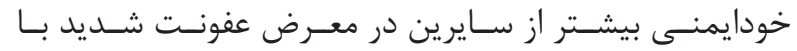

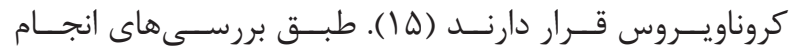

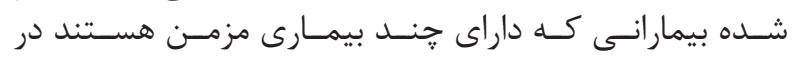

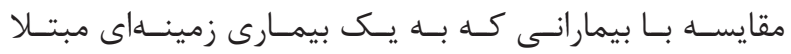

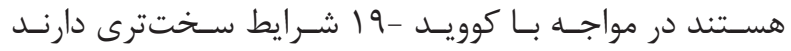

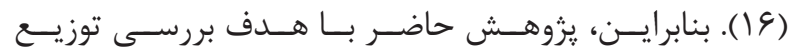

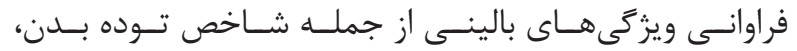

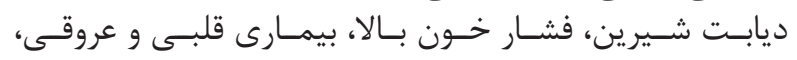

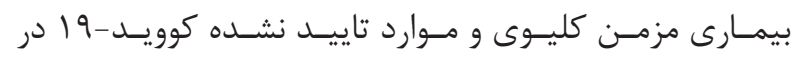

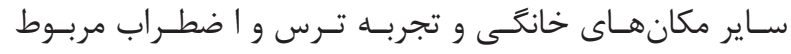

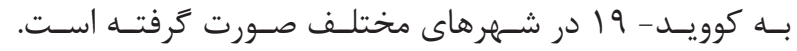

مواد و روشها

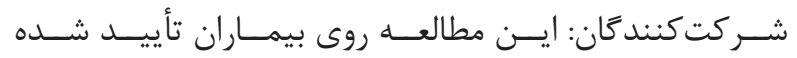

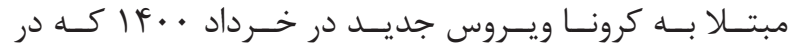

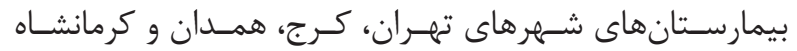

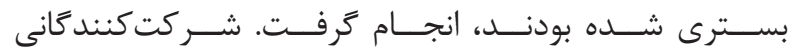

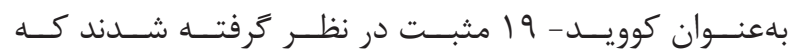

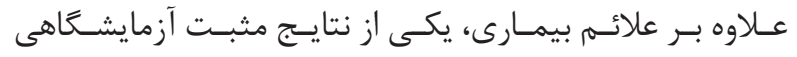

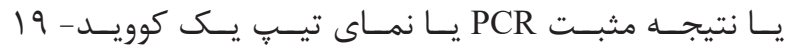

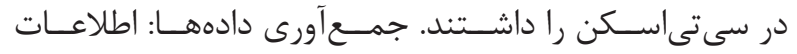

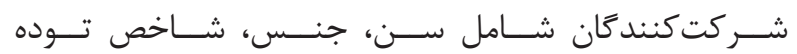

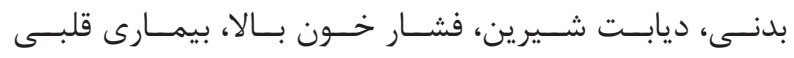

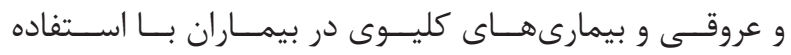

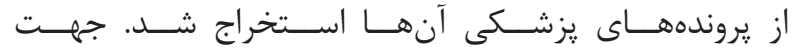

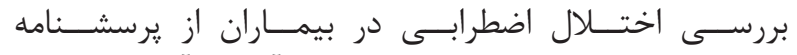

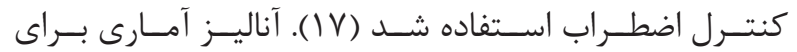

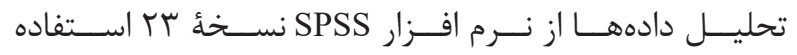

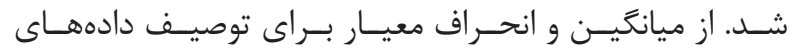

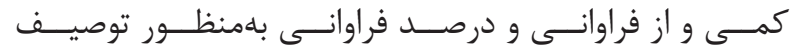

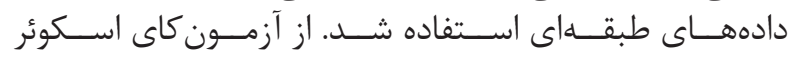

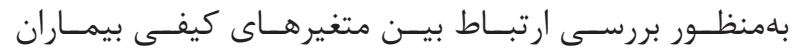

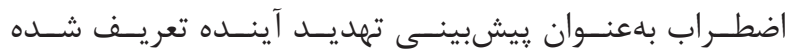

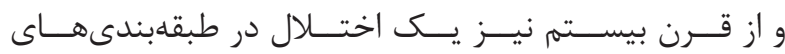

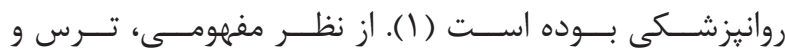

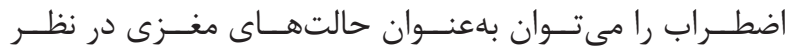

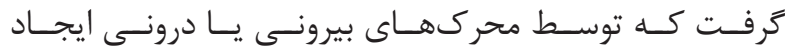

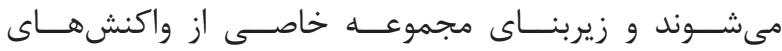

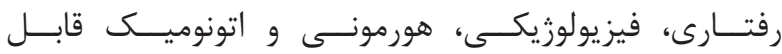

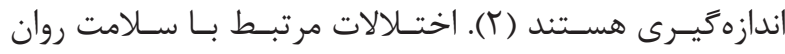

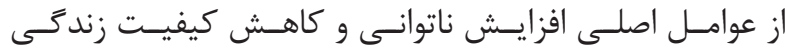

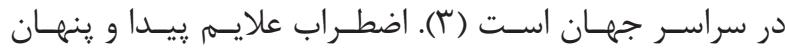

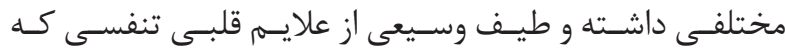

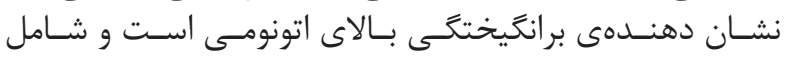

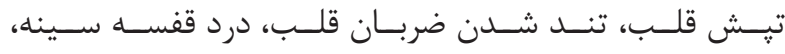

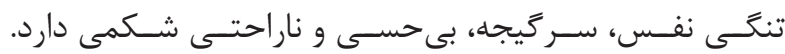

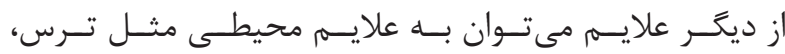

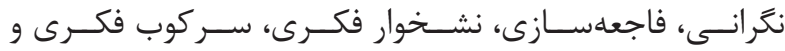

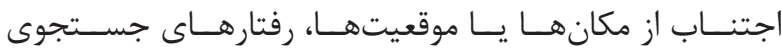

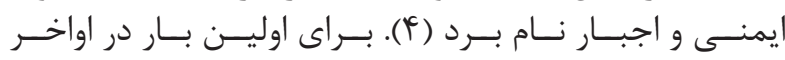

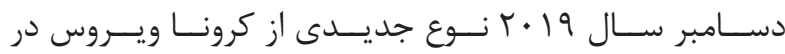

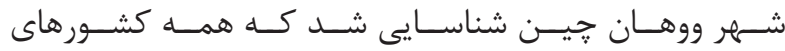

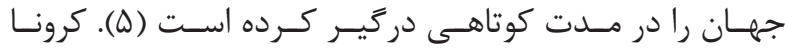

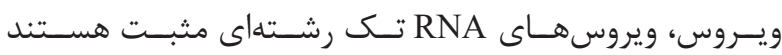

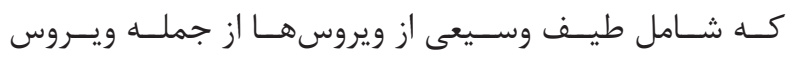

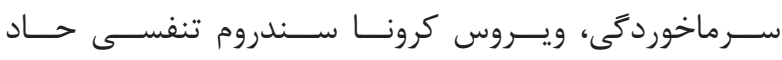

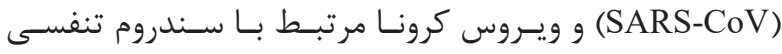

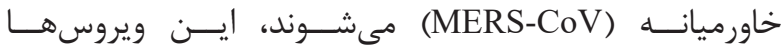

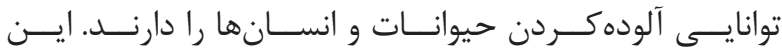

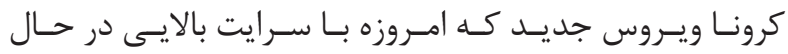

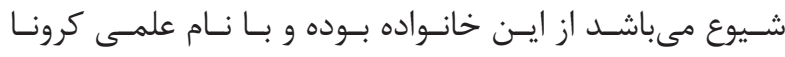

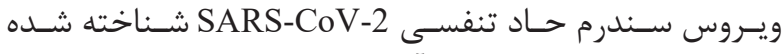

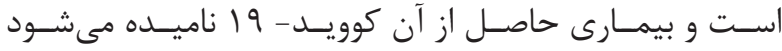

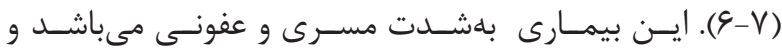

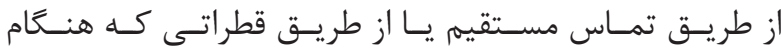

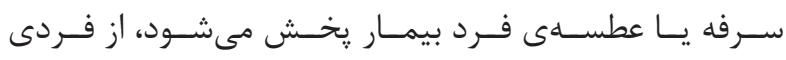

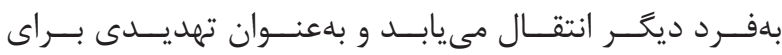

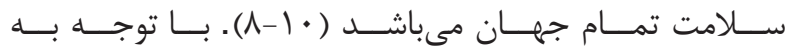

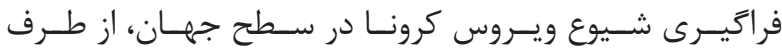

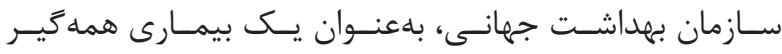

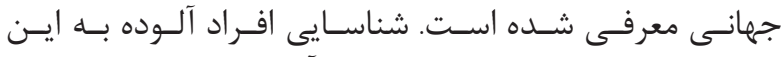

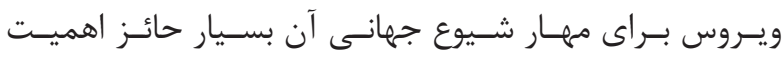

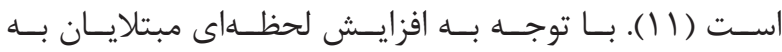

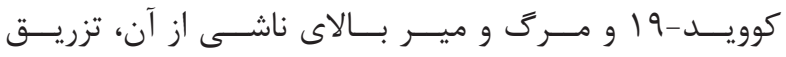

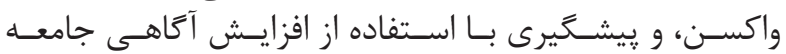




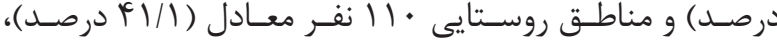

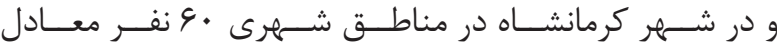
ه دV/V)

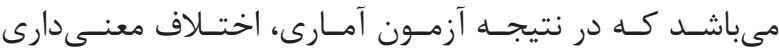

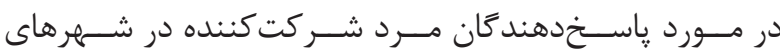

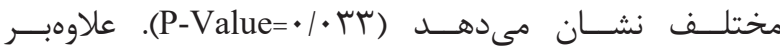

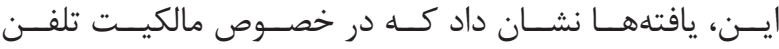

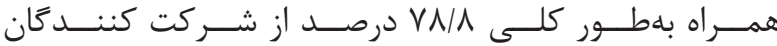

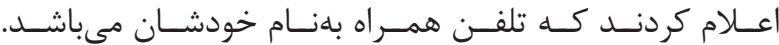

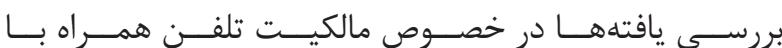

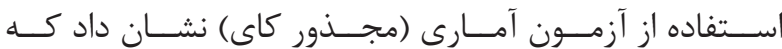

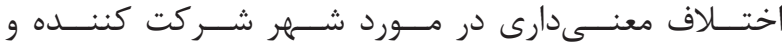

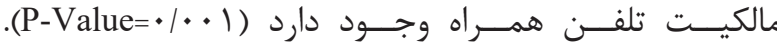

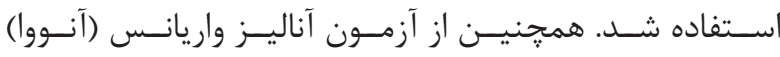

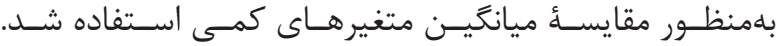

يافته ها

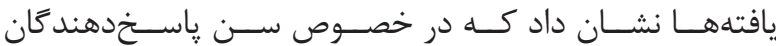

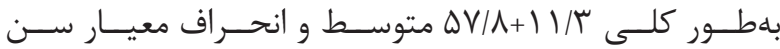

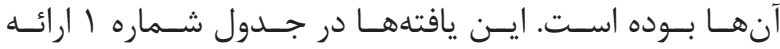

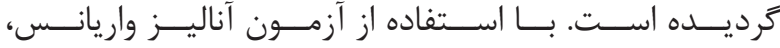

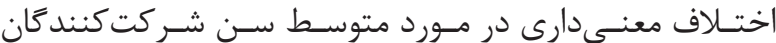

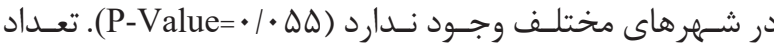

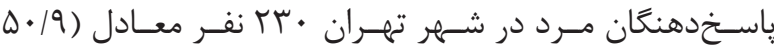

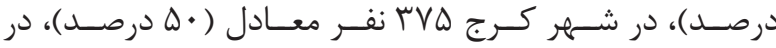

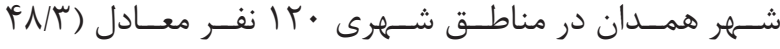

جدول ا- توزيع فراوانى ويزّكىهاى جمعيت شناختى و مقايسٔ آن در شهرهاى مختلف

\begin{tabular}{|c|c|c|c|c|c|c|c|c|c|}
\hline \multirow{2}{*}{ P-Value } & \multicolumn{2}{|c|}{ كرماششاه (Y.r) } & \multicolumn{2}{|c|}{ 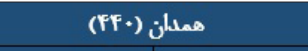 } & \multirow{2}{*}{ كرج (AV·) } & \multirow{2}{*}{ تهران (IFIA) } & \multirow{2}{*}{ مجموع (YIA } & \multirow{2}{*}{\multicolumn{2}{|c|}{ عنوان }} \\
\hline & شهرى (11) & روستايى (Aه) & شهرى (FF) & روستايى (1A) & & & & & \\
\hline$\cdot / \cdot \Delta \Delta$ & $\Delta 9 / 11+N / 9$ & $\Delta V / 1 \cdot+l / T$ & $\Delta N / 1 \cdot+\Delta / \Lambda$ & $\Delta g / 11+g / F$ & $\Delta V / 11+\Delta / \varphi$ & $\Delta V / 11+N / \cdot$ & $\Delta V / 11+N / r$ & سن يالسخدهندكان & ميأئير \\
\hline.$/ 4 r$ & $4 \cdot(\Delta V / / V)$ & $\Lambda \Delta(F \gamma / / 9)$ & $I Y \cdot(F+/ / A)$ & $11 \cdot(f \mid / / 1)$ & $r v \Delta(\Delta \cdot \% \cdot)$ & $r r \cdot(\Delta \cdot / / q)$ & $१ \wedge \Delta(F \uparrow / / \vee \vee)$ & خغن & \\
\hline$<\cdot|\cdot \cdot|$ & $1 \cdots(9 r / / 1 /)$ & $94(\mathrm{VQ} / / \mathrm{r})$ & $1 f \cdot(9 \mid / / T)$ & $1 \Gamma \cdot(\Delta \varphi / / \Delta)$ & $F \mid 9\left(\mathrm{~V} / / / \Gamma^{\prime}\right)$ & $\Delta \cdot r(9 Y / / 9)$ & $10 T Y(Y A / / / \Lambda)$ & 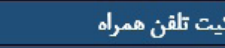 & \\
\hline$<\cdot \mid \cdot \cdot 1$ & $19(9 \cdot / / 1)$ & $\Delta \varphi(I V / / V)$ & $V Y(T F / / 9)$ & $r A(V / / V)$ & $r F \cdot\left(T \Delta / / / T^{q}\right)$ & $f \cdot 9(\mathrm{~V} / / / \mathrm{r})$ & 199(FYT/V) & ازز إيتررثة/لعسترسى & السمغاد \\
\hline \multirow{4}{*}{$<\cdot / \cdot \cdot \mid$} & $F \Delta(11 / / 19)$ & $r q(F \Lambda / / F)$ & $V Y(Y \cdot / / F)$ & $\Lambda \Gamma(T V / / r)$ & $\Delta I(V / / \pi)$ & $9 \Delta(1 \cdot \% / / \cdot)$ & $9 \cdot r(19 / / 9)$ & كم سواد & \multirow{4}{*}{ تمصيلات } \\
\hline & $19(9 / / / V)$ & rq(1F//9) & $r \Delta(9 / / \cdot)$ & $\mid \Lambda\left(F^{2} / / \Lambda\right)$ & $F \wedge(\Lambda / \lambda)$ & $10(Y / /))$ & $r V \Lambda(8 / / 99)$ & ديستان & \\
\hline & $1 \cdot A(F r / / T)$ & $V Y(T r / / / 9)$ & $M+(F \wedge / / T)$ & $1 F \wedge(94 / / 9)$ & Frg(VQ//R) & $r \Lambda r(\Delta \cdot \% / / 9)$ & 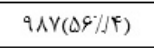 & مدرسه راهنماييى & \\
\hline & $99($ (ัN//9) & $9(\Gamma / / 1)$ & $\Delta r\left(r^{\prime} / K\right)$ & $11(r / / H)$ & $\Delta \Lambda(9 / / \pi)$ & $I V \cdot(r \varphi / / \Delta \Delta)$ & $F \Delta \wedge(19 / / V)$ & كالج و بالاتر از آن & \\
\hline \multirow{3}{*}{$<\cdot / \cdot \cdot 1$} & $19(\Delta / / / r)$ & $V \Delta(\Delta \uparrow / / / 9)$ & $\operatorname{Fr}(10 / / 9)$ & $111(F \Delta / / / 9)$ & KFY(FV//G) & $9 Y(11 \% / / 9)$ & $\Delta 99(\Gamma 1 / / 1)$ & $<F \ldots \ldots$ & \multirow{3}{*}{ مرآمه خاثوار } \\
\hline & $1 \cdot r(F \mid / / 1 \Lambda)$ & $V Y(T r / / T)$ & $110\left(F^{*} \wedge / / / \Gamma\right)$ & $\Lambda 9(Y \varphi / / \Lambda)$ & rDQ(FA//T) & $11 \Delta \Delta\left(\Gamma^{\prime} 9 / / \pi\right)$ & 19V(FY/T) & $f \cdots \cdots \cdot-\gamma \cdots \cdots$ & \\
\hline & $1 \Gamma \Delta(\Delta V / / V)$ & $1 A(\varphi / / r)$ & $9 \Delta(T \Delta / / 9)$ & $F Y(1 K / / 9)$ & $r \varphi(\varphi / / T)$ & $r 9 \Delta\left(F \wedge / / F^{\mp}\right)$ & $991(\mathrm{YF} / / 99)$ & $>v \ldots \ldots$ & \\
\hline \multirow{4}{*}{$<\cdot|\cdot \cdot|$} & $1 \cdot Y(\Delta Y / / V)$ & $\mathrm{Vq}(\mathrm{FF} / / \mathrm{A})$ & $\mid f \cdot(G F / / T)$ & $1 F \wedge(9 \Delta / / 19)$ & $r \wedge q\left(\Delta \Delta / / r^{\top}\right)$ & $r q V(\varphi \cdot / / r)$ & $1 . \Delta \Lambda(\Delta V / / / 9)$ & بيكار & \multirow{4}{*}{ الشتعال } \\
\hline & $r \Delta(q / /)$ & $F 1(19 / / \pi)$ & $r q(11 / / F)$ & $F \mid(I F / / F)$ & $119(Y \Delta / / V)$ & $r A(\varphi / / \cdot)$ & $r 99(10 / / 1)$ & مبتدى & \\
\hline & $V \Delta(r \mid / / V)$ & $\Lambda r(r \Delta / / 9)$ & $91(T+/ / 4)$ & $\Delta r(r \cdot / / 1)$ & $11 \cdot\left(1 \Lambda / / /{ }^{\top}\right)$ & $10 Y(Y Y / / T)$ & $0 \cdot Y(Y \varphi / / r)$ & آموزش ديده/ ماهر & \\
\hline & $\Lambda(1 / / 4)$ & $r(\cdot / / \cdot)$ & $\Delta(\cdot \% / / \cdot)$ & $9(\cdot / / \cdot)$ & $1 Y(\cdot / 9)$ & $\mid V(1 / / / F)$ & $19(\cdot / / / V)$ & كارمند & \\
\hline
\end{tabular}

جدول r- توزيع فراوانى ويزَّىهاى بالينى و مقايسة آن در شهرهاى مختلف

\begin{tabular}{|c|c|c|c|c|c|c|c|c|c|}
\hline \multirow{2}{*}{ P-Value } & \multicolumn{2}{|c|}{ كرمالثاه (T) } & \multicolumn{2}{|c|}{ (ff.) (همان } & \multirow{2}{*}{ (AV.) كر } & \multirow{2}{*}{ تهران (Ifi(A) } & \multirow{2}{*}{ مجموع (YIT) } & \multirow{2}{*}{\multicolumn{2}{|c|}{ عنورن }} \\
\hline & شهرى (il) & روبستايى (Aه) & شهرى (IF.) & روستائي (IA) & & & & & \\
\hline$<. / .+1$ & $r \varphi(f / T)$ & $F r / f+T / f$ & $r N \mid \Delta+\Delta / f$ & $+9 / 0+9$ & $r \Delta / f+q / q$ & $F N / \Delta+T / I$ & $\Delta Q / \Delta+V / 1$ & \multicolumn{2}{|c|}{ شأحص تولهد بلن (BMII) } \\
\hline$<\cdot|\cdot|$, & $\operatorname{lov}(/ 9 \Delta / f)$ & $\operatorname{Gr}(/ . / \Delta / \Delta)$ & $9 F(/ .45 / \Lambda)$ & $f V((1.19 / \mathrm{V})$ & $\operatorname{rgA}(/ . \Delta 1 / 9)$ & $r \cdot 1(/ / f 1 / 9)$ & $\Lambda \Delta \varphi(/ / F Y / \Lambda)$ & \multicolumn{2}{|c|}{ ديالبت شيرين } \\
\hline$<\cdot / . \cdot 1$ & $99(/ / Y / 9)$ & $\operatorname{cr}(/ . \mathrm{V} / \mathrm{T})$ & $\operatorname{lrV}(/ . \Delta \mathrm{V} / \mathrm{V})$ & $199(/ V / V / V)$ & $r f r(/ f r / \Delta)$ & $r \pi r(/ . f V / \cdot)$ & $99 \wedge(/ \Delta 9 / \Gamma)$ & \multicolumn{2}{|c|}{ فشار خهن بالا } \\
\hline$<\cdot \mid \cdot, 1$ & $F 9(/ .10 / f)$ & $r T(/ .1+/ \%)$ & $F A(. I V / f)$ & $99(/ . T V / T)$ & $\Delta r(/ Y / V)$ & $f(1 / 1 \cdot / 9)$ & TFT(JITIT) & \multicolumn{2}{|c|}{ بيمارى قابى و عروقى } \\
\hline$<+1 . .4$ & $\operatorname{Ir}(/ \% / \pi)$ & $10(/ / \% / 9)$ & $f(/ .1 / \cdot)$ & $\mid V(/ / \Delta / V)$ & $9(/ / 1 / T)$ & $1 r(/ / \pi / 1)$ & $\Delta r(/ / Y / \Delta)$ & \multicolumn{2}{|c|}{ بيمارى مزمن كايوى } \\
\hline .114 & $\mathrm{~V}(/, / \mathrm{r} / \mathrm{f})$ & $\Delta(/ / . / /)$ & $1(1 \cdot \cdot / 0)$ & $f(. / 1 \cdot)$ & $r(1 \cdot \cdot(4)$ & $9(1 . \cdot 19)$ & $r \Delta(/ .1 \cdot)$ & \multicolumn{2}{|c|}{ بيمارى مزمن أسـاداد ريوى } \\
\hline & ro & 191 & 15. & $r 1$. & Av. & $1 f 10$ & TATA & \multicolumn{2}{|c|}{ 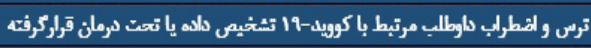 } \\
\hline & $12(7.99)$ & $v 9(/ . \& \Delta)$ & $V A(/ .9 \cdot)$ & $10 \Delta(/ \Delta \Delta)$ & $F \mid \wedge(\mid F \lambda)$ & $9 \times 9(/ . \mathrm{F \Delta}$ & $1 \Gamma \lambda \cdot(/ . \mathrm{F} / \mathrm{T})$ & \multicolumn{2}{|c|}{ بسترى در ييمارستان كويد - 19} \\
\hline & بين 4 تا اروز & بين 4 تا 9 وروز & بين \نا ·اروز & بين V تا و روز & بين 4 تا · اروز & بين 4 تا · اروز & بين 9 تا 9 روز & \multicolumn{2}{|c|}{ تعداد روز بستركى متوسط (IQR) } \\
\hline.$/ 114$ & To & $\Delta T$ & 91 & 1.5 & rif & $r 49$ & 191 & \multicolumn{2}{|c|}{ تشخيص و درمان - خائواده برائ كويد- 19} \\
\hline$<\cdot / \cdot \cdot 1$ & $1 \cdots(/ A Y / \Delta)$ & $1 \pi r(/ 9 \% / 1)$ & $11 \cdot(/ \Delta f / V)$ & $111(/ . \Delta \Gamma / 1)$ & $f \cdot 1(/ . A Y / T)$ & $r \varphi \Delta(/ 91 / 9)$ & $119 r(/ 9 N \mathrm{VN})$ & \multicolumn{2}{|c|}{ 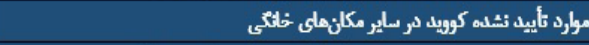 } \\
\hline$\langle\cdot| \cdot \cdot 1$ & $\Lambda \wedge(/ 9 \Delta / \Lambda)$ & $1 T f(/ / 99 / \Lambda)$ & $1 \%(1 / 9 \Delta / r)$ & $r \cdot \Lambda(/ .99 / \Delta)$ & $1 F A(I / . \cdot \%)$ & rev(/.9T/1) & $1+\mathrm{T}(\mathrm{(/. \Delta 9/T)}$ & \multicolumn{2}{|c|}{ تجوريه ترص و الفطراب مريوط به كويده- 19} \\
\hline.$/ 1 \mathrm{r}$ & $\cdot(\% \cdot \%)$ & $\cdot(\% \cdot \%)$ & $1(1 \cdot / \theta)$ & $f(/ /, / 9)$ & $r(\cdot / \uparrow)$ & $A(/ / / 9)$ & $10(\% \cdot / 9)$ & \multicolumn{2}{|c|}{ باترين تجروه مرتبط با كويد - 19} \\
\hline \multirow{4}{*}{$<\cdot . \cdot 1$} & $r A(/ 1.1 \%)$ & $9(/ / r / 1)$ & fr(/.MNf) & $\Delta r(/, T / / 1)$ & $r \Delta(/ .1 T / \Lambda)$ & $\Delta \varphi(/ .1 V / 9)$ & $r 4 \cdot(/ .1+19)$ & خيلى خوب & \multirow{4}{*}{ مقابله با السترك در طى كويد- 19} \\
\hline & $99(/ / f 1 / r)$ & $11+(/ F V / 9)$ & $\operatorname{IVr}(/ \mathrm{VN} \mid \bar{\varphi})$ & $\operatorname{1Ar}(/ \mathrm{YNQ} \Delta)$ & IV9(//.rT/4) & $r \cdot \Delta(/ f f f / T)$ & $१ \wedge q(/ . ศ q / \cdot)$ & bugn & \\
\hline & $\Delta 9(/ . / \Delta / \varphi)$ & $\operatorname{Ar}(/ .99 / 0)$ & $\begin{array}{r}(/ . / Y \cdot) \\
\end{array}$ & $\mathrm{r}(\mathrm{H} \cdot \%)$ & 9.(INII) & $11 \cdot(/ r)(9)$ & $r q+(.19 / T)$ & بامشكل & \\
\hline & $r \Delta(/ .1 T / \Delta)$ & $f((/ .1 T / \Delta)$ & $f(/ .1 / \cdot)$ & $r(1 \cdot \cdot \Delta)$ & $\operatorname{rfv}(/ f \Delta \Delta \mathrm{V})$ & $9 \cdot(.1 .91 \%)$ & $0 \cdot r(1 . \cdot+1)$ & بلانون السترس & \\
\hline
\end{tabular}




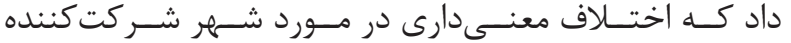

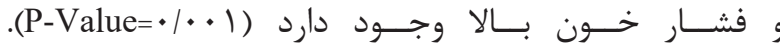
يافتههــا نشـان داد كـهـ در خصـوص تسـت قنــد خــون ناشـتا

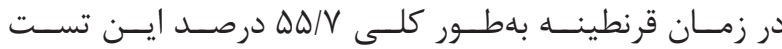

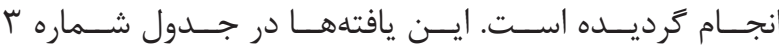

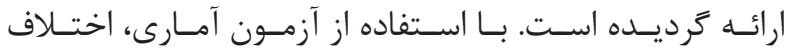

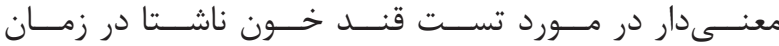

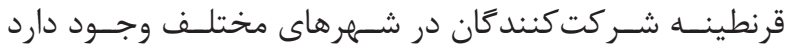

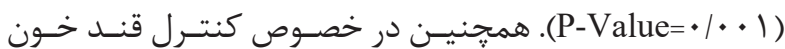

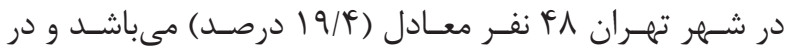

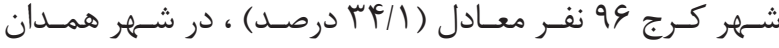

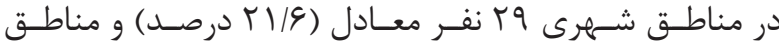

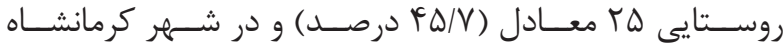

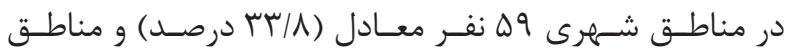

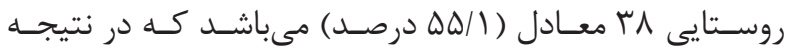

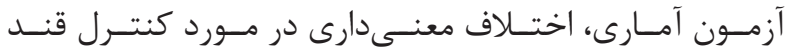

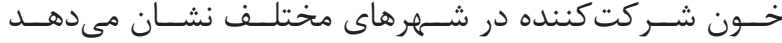

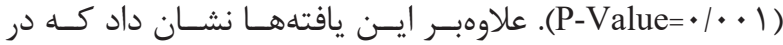

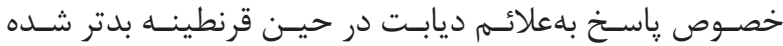

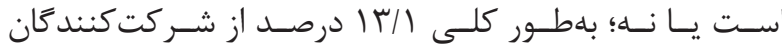

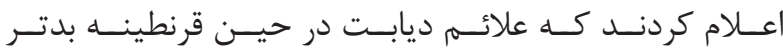

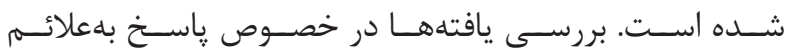

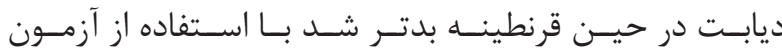

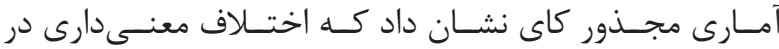

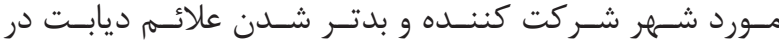

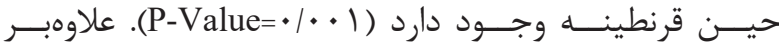

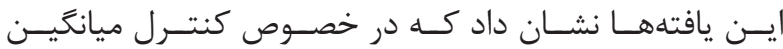

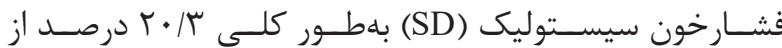

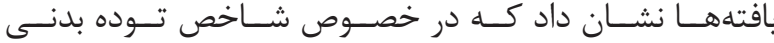

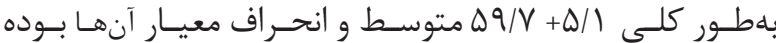

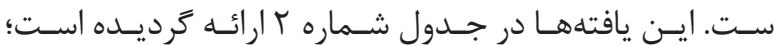

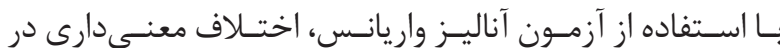

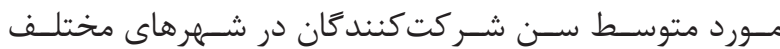

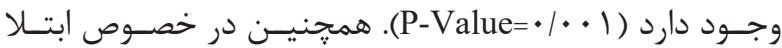

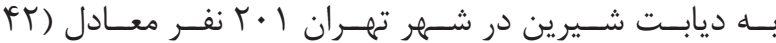

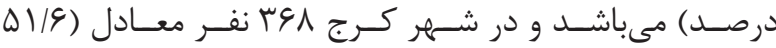

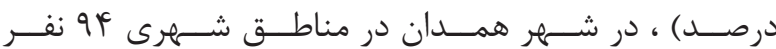

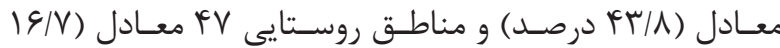

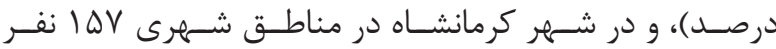

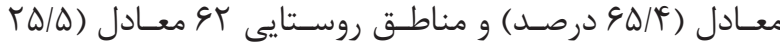

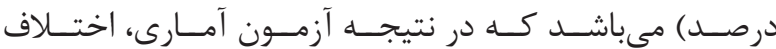

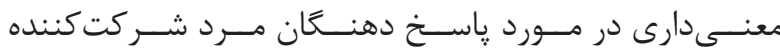

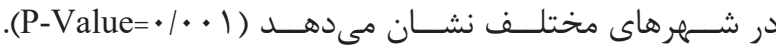

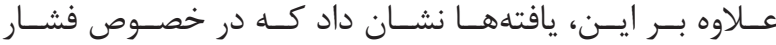

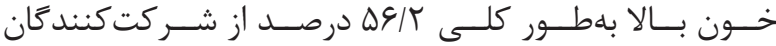

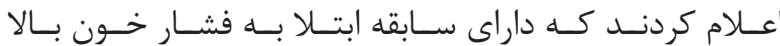

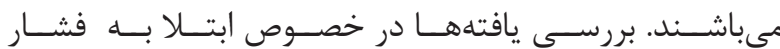

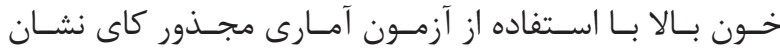

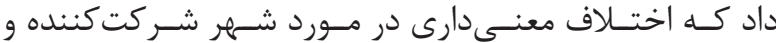

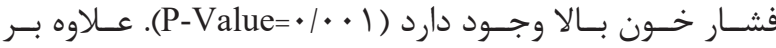

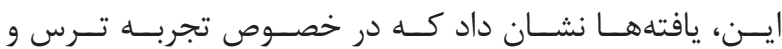

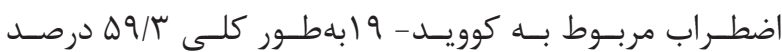

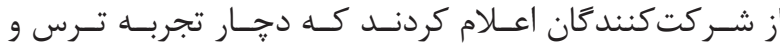

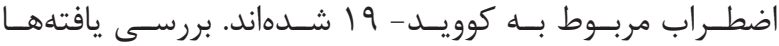

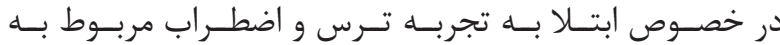

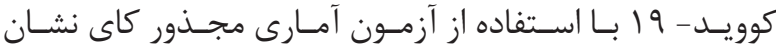

جدول بـ- توزيع فراوانى دسترسى به مراقبهاى بهداشتى و مقايسأ آن در شهرهاى مختلف

\begin{tabular}{|c|c|c|c|c|c|c|c|c|}
\hline \multirow{2}{*}{ P-Value } & \multicolumn{2}{|c|}{ كرماتشاه (T· (r) } & \multicolumn{2}{|c|}{ (ff.) همدان } & \multirow{2}{*}{ كأ (AV.) } & \multirow{2}{*}{ تهران (Ifis) } & \multirow{2}{*}{ مجموق (TYYA) } & \multirow{2}{*}{ عنون } \\
\hline & شهرى (IIA) & روستايى( & شهرى (F.) & روستائي (1A) & & & & \\
\hline & 119 & $v_{\Delta}$ & 11. & $9 \lambda$ & for & TAY & 1.19 & ييامد سلاهتى در افراد ديالجَى (N) \\
\hline$<+. .1$ & $19(/ F N \Delta)$ & $\pi r(/ . T F / V)$ & $\Delta r(/ F T / \cdot)$ & $r A(/ . T V / 1)$ & $\operatorname{lVr}(/ . \Delta 9 / T)$ & $\operatorname{IVA}(/ \mathrm{VT} / T)$ & $9 \lambda \cdot(/ \Delta \Delta / V)$ & 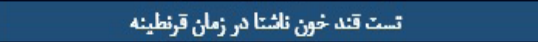 \\
\hline$<+. . .1$ & ro(/.१/9) & $\cdot(\%+\%)$ & $11(/ / / \Delta)$ & $\cdot(/ . \cdot \cdot \cdot)$ & $1 \cdot(/ . / 1)$ & $\mid N(/ . \Delta / 9)$ & $\Delta r(/ / F / V)$ & تسك HbAlC در طول قرنطينه \\
\hline \multirow[t]{3}{*}{$<\cdot . \cdot 1$} & $110(f f / r)$ & $V \Delta(/ . / T / f)$ & $\mid A T(/ . T T / 1)$ & $I V \cdot(/ . \Lambda T / \varphi)$ & rfo(/.AT/I) & $\pi r \cdot(/ .9 f / Q)$ & $f \Delta r(/ . / / / A)$ & 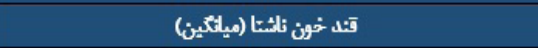 \\
\hline & $19(.1 \% / r)$ & $11(/ .+Y / 9)$ & $g(T \cdot)$ & $r(/ .19 / V)$ & $\pi r(/ .19 / 9)$ & $r(/ .19 / \pi)$ & $\operatorname{fr}(/ / 1 V / 9)$ & 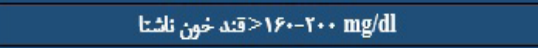 \\
\hline & $f(/ / T / \Lambda)$ & $9(7 / T N 9)$ & $1(\cdot)$ & $9(/ A T / T)$ & $f r(/ / T / / \Delta)$ & $r q(/ .1 \% / \Delta)$ & $99(/ 19 / r)$ & 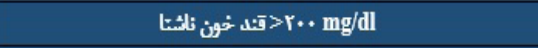 \\
\hline$<+. \cdot 1$ & $\Delta 9(/ / T / \Lambda)$ & $r A(/ \Delta \Delta / 1)$ & $r q(/ / T / / 9)$ & $r \Delta(/ f \Delta / Y)$ & $99(/ / T 4 / 1)$ & $f \wedge(/ .1 / / f)$ & ror $(/ / r 1 / *)$ & كتبرل قَند خُخون \\
\hline \multirow[t]{5}{*}{$<+1 . .1$} & $r \cdot(/ 11 / / /)$ & $11(.199 T)$ & $10(/ .1 \cdot / \pi)$ & $\mid 1 T(/ T T / 9)$ & $r \Delta(/$. / $\cdot)$ & $F 9(/ .1 N T)$ & $1 f \cdot(/ .1 T / 1)$ & 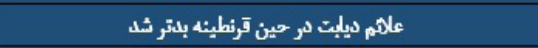 \\
\hline & $11(/ .0 / 9)$ & $r(/ T / \cdot)$ & $19(/ . M N)$ & $\Delta(/ . \Delta / V)$ & $\mid V(/ . \Delta / 1)$ & $\Delta r(/ r r / 9)$ & $99(/ 11 / T)$ & يكبار در ماه \\
\hline & $111(/ .1 \cdot / 9)$ & $95(/ 9 \% / 9)$ & $\operatorname{Ar}(/ / . \cdot / \Delta)$ & $f \vee(/ 9 f / r)$ & $r \Delta G(J / Y N(\Delta)$ & 19(/.TN9) & $99 \lambda(/ / V / / r)$ & كتبرل نشدن كلوكز در خانه \\
\hline & 1.0 & 99 & 10. & $1 \%$. & $r \Delta \Lambda$ & rel & $9 V_{\Delta}$ & ع عوارض دراقراد مبتلا به فشارخون (N) \\
\hline & $9 \cdot(/ \Delta N / T)$ & $r \Delta(/ / r+/ 9)$ & $\operatorname{Ar}(/ . T /(\Delta)$ & $9 \cdot(/ f \Delta / \Delta)$ & $\operatorname{frA}(/ \cdot \cdot \cdot / \Delta)$ & $19 \Delta(/ 9 V / \Lambda)$ & $\Delta r \cdot(/ \Delta r / \Lambda)$ & أندازهيري فُشار خون در قرنطينه \\
\hline$<+1 . .1$ & $r(/ .1 f / T)$ & $r \cdot(/ .19 / 0)$ & $19(/ .19 / r)$ & $r t(/ . r q / Y)$ & $1 r \Delta(.19 / 4)$ & $9 \Delta(/ .1 N /)$ & $r q \cdot(l . r \cdot / r)$ & فشارخون سبستوليك، مبانئين (SD) \\
\hline \multirow[t]{2}{*}{$<\cdot \%+4$} & $\Delta A \mid g(|Y| I)$ & $\Delta F(A(/ N N T)$ & $\Lambda \Delta / \Lambda(/ / V / \cdot)$ & $91 / A(/ . T \Delta / 9)$ & $\Delta A / 9(/ 11 / \cdot)$ & $\operatorname{ANr}(/ 11 / 1)$ & $19 / 9(/ .1 \Gamma / 0)$ & 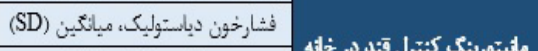 \\
\hline & A.('MN9) & $99(/ .19 / T)$ & $19 \lambda(/ . Y \Delta) \cdot \cdot)$ & $9 \cdot(/ .09 / 4)$ & $\operatorname{rqV}(/ / 9 V / 9)$ & Tfr(/g9/9) & $r \Lambda \cdot(/ Y Y / 9)$ & $\mathrm{SBP}<140 \mathrm{mmHg}$ \\
\hline \multirow[t]{2}{*}{$<\cdot, \cdot 1$} & A(I/N9) & $r(/ 1 \cdot / r)$ & $9(/ .19 / V)$ & $9(/ / T / 1)$ & $\mid r(/ . / Y / \uparrow)$ & $19(.19 / 1)$ & $\Delta V(/ .1 \Delta / V)$ & SBP>140-160 mmHg \\
\hline & $\begin{array}{ll}(/ \pi / \pi) \\
\end{array}$ & $9(/ / / / \mathrm{f})$ & $\Delta(/ N T)$ & $1 \cdot(/ / \mathrm{r} \cdot / \Delta)$ & $\lambda(/ .1 \cdot / V)$ & $10(/ .1 \% / T)$ & $r \Delta(/ .9 / 9)$ & $\mathrm{SBP}>160 \mathrm{mmHg}$ \\
\hline$\angle+1 \cdot \pi r$ & $9(\Delta / 9)$ & $\Gamma I(.1 T / \Lambda)$ & $\Delta(/ / F / \Lambda)$ & $r f(/ 1 \Delta / 9)$ & $9 \Delta(/ / T) / \cdot)$ & $1 A(/ / N 9)$ & $1 f r(/ 1 f / 9)$ & فُشار خون كنترل شده \\
\hline$<+., 1$ & $\operatorname{Ir}(/ / N \Delta)$ & $1 r(/ .19)$ & $A(/ . V Y Y)$ & TAC/TF/Y & $9(/ / / 9)$ & $\operatorname{Fr}(/ / \cdot \cdot / \Lambda)$ & $1 T \cdot(/ .1 T / T)$ & افرايش فشار خون در قرنطينه \\
\hline
\end{tabular}




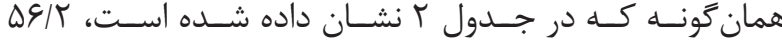

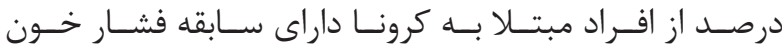

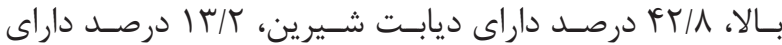

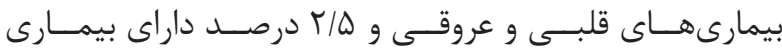

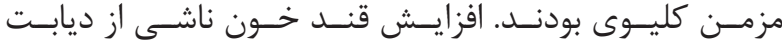

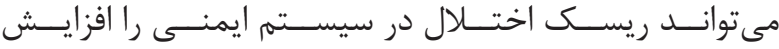

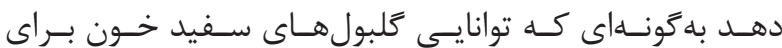

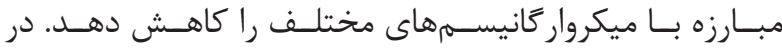

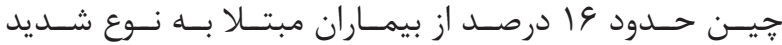

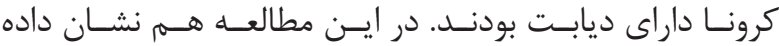

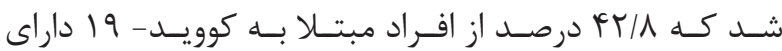

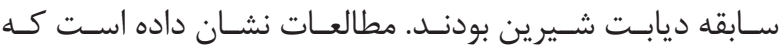

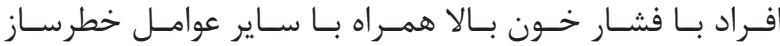

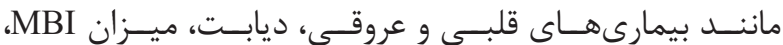

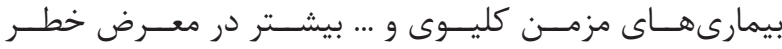

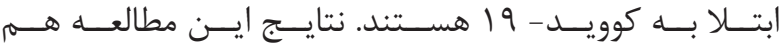

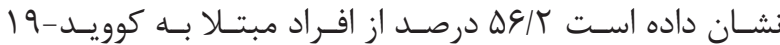

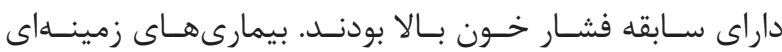

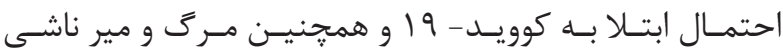

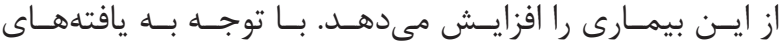

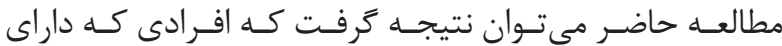

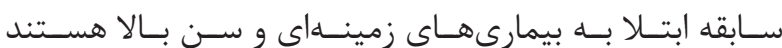

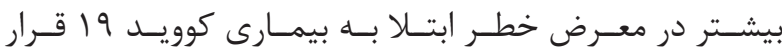

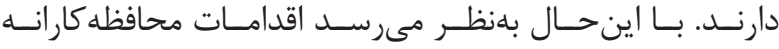

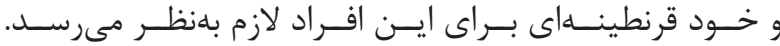

1. Crocq MA. A history of anxiety: from Hippocrates to DSM. Dialogues Clin Neurosci. 2015 Sep;17(3): 319-25.

2. Tovote P, Fadok JP, Lüthi A. Neuronal circuits for fear and anxiety. Nat Rev Neurosci. 2015 Jun;16(6):317-31.

3. Yohannes AM, Alexopoulos GS. Depression and anxiety in patients with COPD. Eur Respir Rev. $2014 \quad$ Sep;23(133):345-9.

4. Tully, P.J, Harrison, N.J, Cheung, P. Anxiety and Cardiovascular Disease Risk: a Review. Curr Cardiol Rep 18, 120 (2016).

5 Huang C, Wang Y, Li X, Ren L, Zhao J, Hu $\mathrm{Y}$, et al. Clinical features of patients infected with 2019 novel coronavirus in Wuhan, China. The Lancet. 2020;395(10223):497-506.

6.Surveillances V. The epidemiological characteristics of an out break of 2019 novel Coronavirus Diseases (COVID-19)-China. China CDC Weekly. 2020; 2(8):113-22.

7. Abdolahi S, Gorji A. COVID-19: The potential role
شــركت كنند

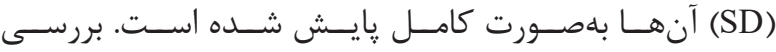

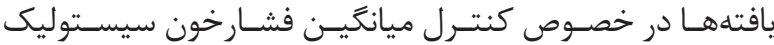

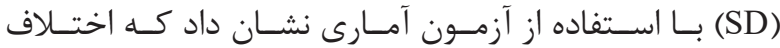

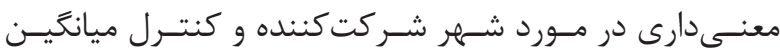

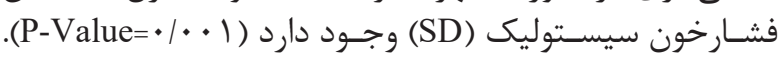
بحث و نتيجه كيرى

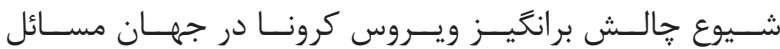

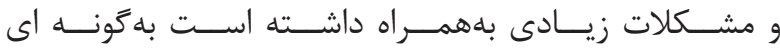

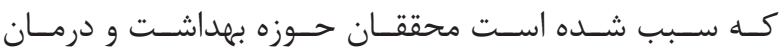

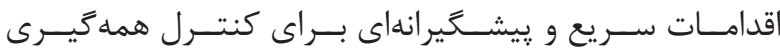

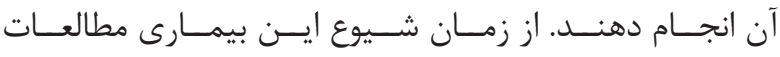

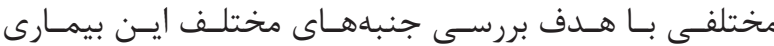

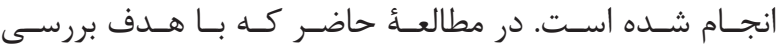

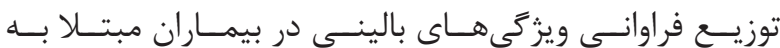

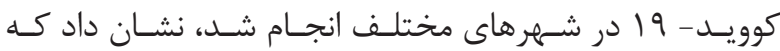

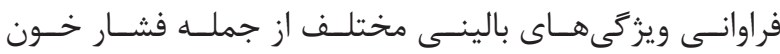

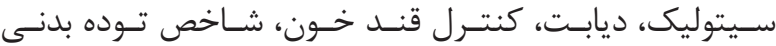

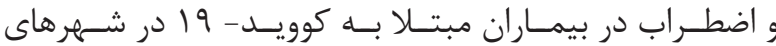

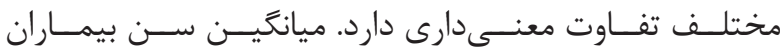

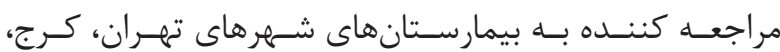

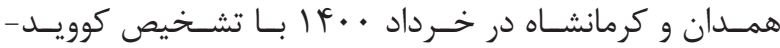

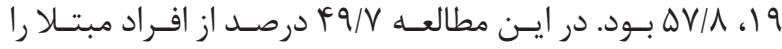

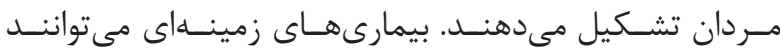

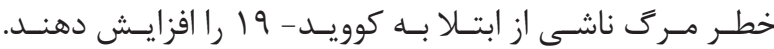

منابع of nutritional deficiencies, global climatic changes, and immune system dysfunction. Iranian Journal of War and Public Health. 2020 Nov 10;12(4): 249-258.

8.Chan JF-W, Yuan S, Kok KH, To KKW, Chu $\mathrm{H}$, Yang $\mathrm{J}$, et al. Afamilial cluster of pneumonia associated with the 2019 novel Coronavirus indicating person-to-person transmission: A study of a family cluster. The Lancet. 2020; 395(10223): 514-23.

9. Dong X, Cao Y-Y, Lu X-X. Eleven faces of coronavirus disease 2019. Allergy. 2020; 75: 1699- 709.

10. MocciaF, Gerbino A, Lionetti V, Miragoli M, Munaron L, Pagliaro P, et al. COVID-19-associated cardiovascular morbidity in older adults: a position paper from the Italian Society of Cardiovascular Researches. JCR 2020: 1-29.

11. Ji Y, Ma Z, Peppelenbosch MP, Pan Q. Potential association be-tween COVID-19 mortality and health-care resource availability. The Lancet Global Health. 2020; 8(4):e480.

12. Ou M, Zhu J, Ji P, Li H, Zhong Z, Li B, et al. Risk 
factors of severe cases with COVID-19: A metaanalysis. Epidemiology \& Infection. 2020; 148: e175.

13. Rahman A, Sathi NJ. Risk factors of the severity of COVID-19:A meta-analysis. International Journal of Clinical Practice. 2020; e13916.

14.Richardson S, Hirsch JS, Narasimhan M, Crawford JM, McGinn T, Davidson KW, et al. Presenting characteristics, comorbidities, and outcomes among 5700 patients hospitalized with COVID-19 in the New York City area. JAMA. 2020; 323(20): 2052-9.

15. Fortin M, Lapointe L, Hudon C, Vanasse
A. Multimorbidity is common to family practice: Is it commonly researched? Canadian Family Physician. 2005; 51(2): 244-5.

16. Gonzalez JS, Safren SA, Cagliero E, Wexler DJ, Delahanty L, Wit- tenberg E, et al. Depression, selfcare, and medication adherence in type 2 diabetes: Relationships across the full range of symptom severity. Diabetes Care. 2007; 30(9): 2222-7.

17. Salari-Moghaddam S, Ranjbar A, Fathi-Ashtiani A. Validity and Reliability measurement of the Persian version of anxiety Control Questionnaire. Journal of Clinical Psychology, 2018; 9(4): 33-43. 